\title{
Trabajo en grupo en base a ejercicios de formato cerrado
}

\author{
SALVADOR CHACÓN-MOSCOSO \\ Universidad de Sevilla \\ Facultad de Psicología \\ Departamento de Psicología Experimental \\ schacon@us.es \\ ORCID: https://orcid.org/0000-0002-6401-7384 \\ D.O.I.: http://dx.doi.org/10.12795/JDU.2018.i01.08 \\ Pp.: $146-160$
}

\section{Resumen}

El Ciclo de Mejora Docente se aplicó en la asignatura Diseño y Análisis de Datos en Psicología I del Grado en Psicología. El objetivo fue variar el sistema de evaluación, lo que llevaría a variar también la metodología: se pasaría de pruebas de formato abierto a formato cerrado. La principal razón era disminuir el tiempo de espera del alumnado de recibir feedback de los resultados obtenidos. El alumnado trabajó en grupos a partir de ejercicios de formato cerrado, llevando en todo momento el peso de la actividad. Con un cuestionario aplicado al inicio y al final del ciclo, se midió el cambio en el conocimiento del alumnado. Los resultados fueron satisfactorios en tiempo de espera de los resultados en las evaluaciones, conocimientos adquiridos, asistencia a clase y participación en el cuestionario. Como trabajo futuro, se espera ampliar los materiales de formato cerrado y trabajar más con el software SPSS. 
Palabras clave: Diseño y Análisis de Datos en Psicología I, Grado en Psicología, docencia universitaria, innovación docente universitaria, formato cerrado.

\section{Descripción del contexto}

El Ciclo de Mejora Docente se aplicó en la asignatura Diseño y Análisis de Datos en Psicología I, de primer curso del Grado en Psicología, segundo cuatrimestre. Las clases se impartían en el grupo A, grupo bilingüe. En esta asignatura específicamente, la docencia se impartió en inglés, y los materiales y exámenes también estuvieron en inglés. Era un grupo de estudiantes educado y cordial, que mostraba interés en las clases.

Aun siendo de primero, el alumnado ya tenía conocimientos básicos del uso del software SPSS que utilizábamos en clase, adquiridos durante el primer cuatrimestre. Encontramos los mayores desajustes con algún alumnado extranjero, que no había utilizado nunca el programa, por lo que le suponía más esfuerzo seguir las explicaciones.

El año pasado se logró integrar más la teoría con la práctica, tanto con ejercicios con calculadora como con software, dando una visión integral a toda la materia (Chacón-Moscoso, 2017). El objetivo para el curso 2017/18 fue variar el sistema de evaluación con respecto al curso anterior, lo que llevaría a variar también la metodología: se pasaría de pruebas de formato abierto (donde el alumnado genera la respuesta) a formato cerrado (donde el alumnado trata de elegir la respuesta correcta de entre varias dadas) (Barbero-García, Vila-Abad y Holgado-Tello, 2010). La principal razón era disminuir el tiempo de espera del alumnado de recibir feedback de los resultados obtenidos.

Jornadas de Formación e Innovación Docente del Profesorado | № 1 (2018) Esta obra se distribuye con la licencia Creative Commons 


\section{Diseño previo del Ciclo de Mejora Docente}

\section{Modelos metodológicos}

El modelo metodológico (de Alba y Porlán, 2017) habitual suponía la impartición de teoría, aplicación de ejercicios de formato abierto por parte del alumnado, y corrección en la pizarra, ya fuera por parte de alumnado voluntario o por el profesor. El modelo metodológico ideal mantendría igual la impartición de teoría y la corrección; sin embargo, plantearía todos los ejercicios de formato cerrado, para que sirvieran de entrenamiento de cara a las pruebas de evaluación. Finalmente, el modelo metodológico posible sería realizar al menos el $25 \%$ del total de prácticas en formato cerrado, dado que realizar pruebas estadísticas de formato cerrado es algo muy costoso: implica no sólo ejecutar los cálculos y repasarlos, sino que además hace falta pensar en buenos distractores (respuestas no correctas), que tiendan a ser respondidas por aquel alumnado que tiene menos conocimiento.

La Figura 1 muestra una representación de estos tres modelos metodológicos (el habitual, el ideal y el que se consideró posible):

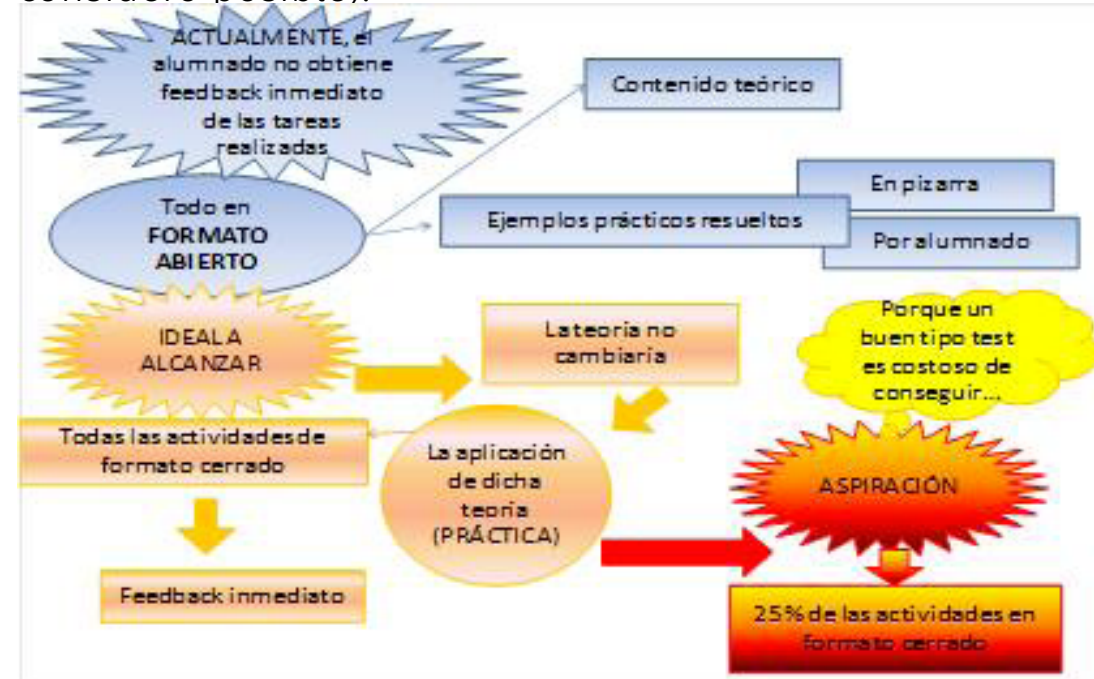

Figura 1. Representación del modelo actual, el ideal y el que se aspira a conseguir.

Jornadas de Formación e Innovación Docente del Profesorado I № 1 (2018)
Esta obra se distribuye con la licencia Creative Commons Reconocimiento-NoComercial-SinObraDerivada $\quad 4.0$ Internacional (CC BY-NC-ND 4.0.) 


\section{Mapa de contenidos y preguntas asociadas}

El mapa de contenidos (García-Díaz, Porlán y Navarro, 2017) se basó en los temas de estadística descriptiva presentes en el programa de la asignatura. La Figura 2 representa dichos contenidos.

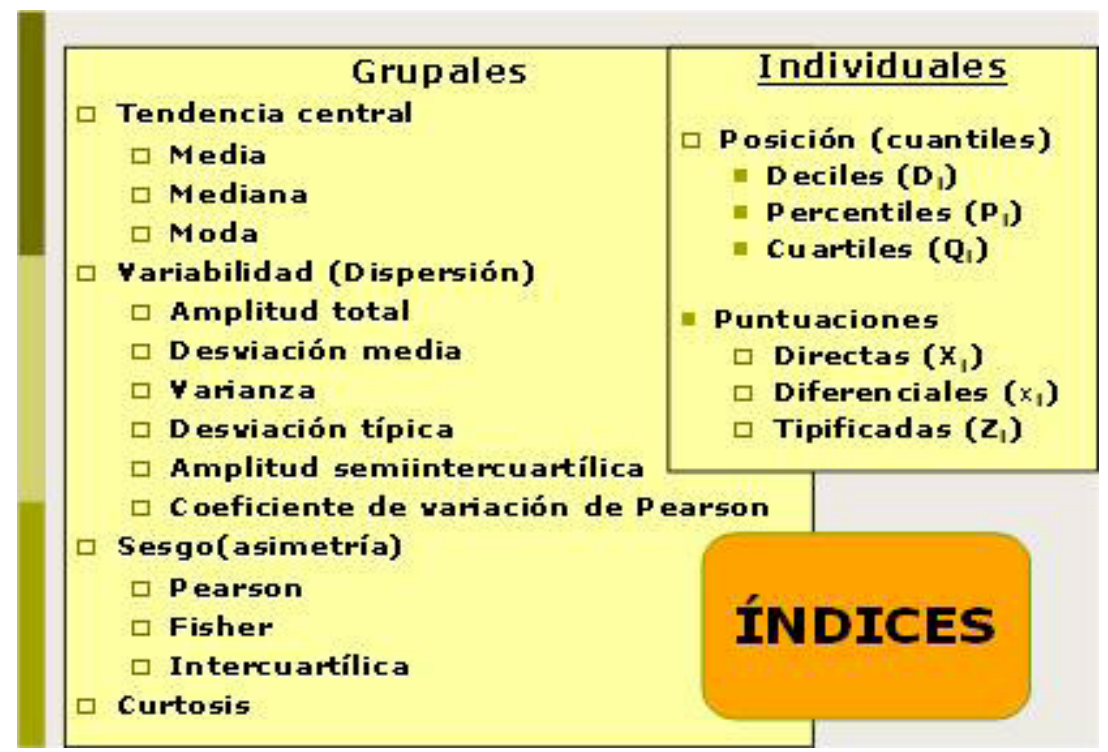

Figura 2. Contenidos a tratar en el ciclo de mejora.

Se presentaron casos con los que había que realizar diferentes aplicaciones, empezando por las más sencillas y terminando por las más complejas. Concretamente, los contenidos que se pretendió que aprendieran (no teóricamente, sino de manera aplicada, en base a ejercicios prácticos) y las preguntas problemas asociados fueron los siguientes:

1. Tipos de variable - ¿Cuáles son los tipos de variables posibles en función de su escala? ¿Cómo puedo distinguir entre ellas?

2. Introducción de variables en SPSS: ¿qué representa una línea en SPSS? ¿Y una columna? ¿Cómo introduzco los datos?

Jornadas de Formación e Innovación Docente del Profesorado | № 1 (2018)

Esta obra se distribuye con la licencia Creative Commons Reconocimiento-NoComercial-SinObraDerivada $\quad 4.0$ Internacional (CC BY-NC-ND 4.0.) 
3. Gráficas: ¿Qué gráficas son apropiadas para cada tipo de variable?

4. Frecuencia relativa: ¿Para qué sirve? ¿Cómo se calcula?

5. Frecuencia relativa acumulada: ¿Para qué sirve? ¿Cómo se calcula?

6. Moda: ¿Para qué sirve? ¿Cómo se calcula?

7. Mediana: ¿Para qué sirve? ¿Cómo se calcula?

8. Media aritmética: ¿Para qué sirve? ¿Cómo se calcula?

9. Diferencias entre moda, media y mediana: ¿cuándo es más conveniente usar cada una de ellas?

10. Percentiles: ¿Para qué sirve? ¿Cómo se calculan?

La Figura 3 representa los contenidos a desarrollar y su relación con las preguntas problemas.

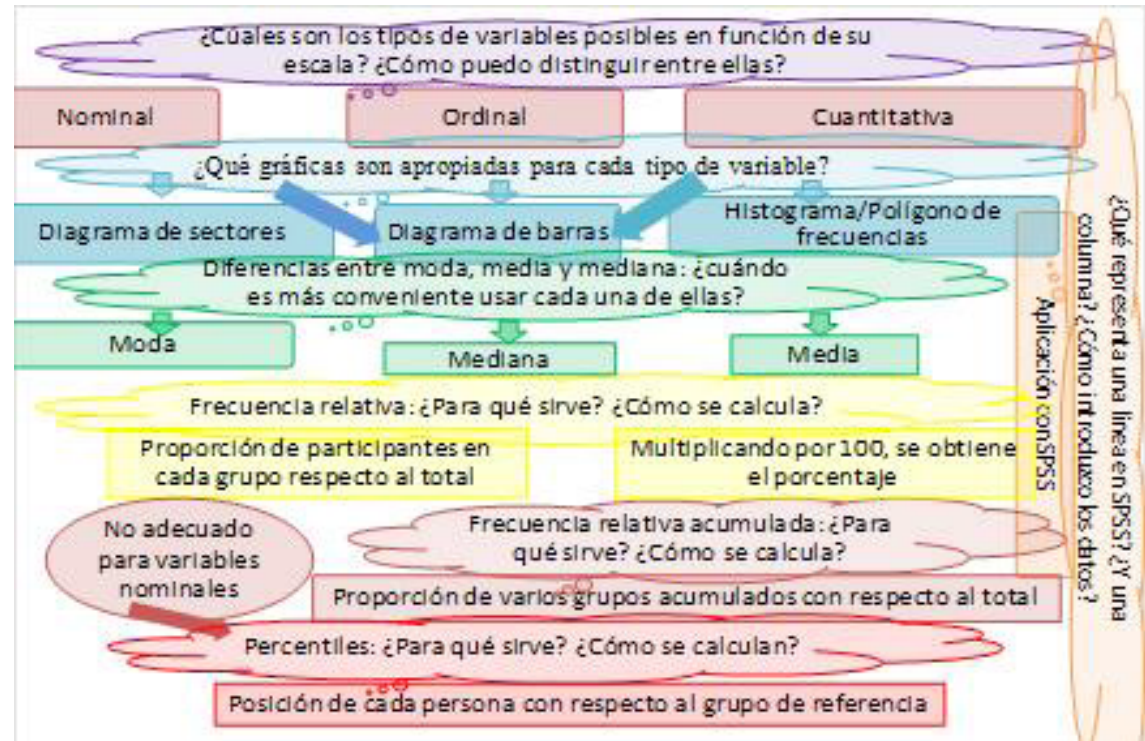

Figura 3. Contenidos a desarrollar y relación con las preguntas problema.

\section{Secuencia de actividades}

Se realizó una secuencia de actividades siguiendo el modelo metodológico "posible" (preguntas de formato cerrado a partir de un caso). Un ejemplo de caso al que se 
aplicaron los 10 contenidos a tratar y que formó parte de ese $25 \%$ de ejercicios de formato cerrado que se pretendía alcanzar en este ciclo de mejora fue el siguiente:

La siguiente tabla presenta, agrupados en intervalos, los años de servicio de los trabajadores en una institución.

\begin{tabular}{|l|l|}
\hline $\begin{array}{l}\text { Años de } \\
\text { servicio }\end{array}$ & fi \\
\hline $0-4$ & 45 \\
\hline $5-9$ & 50 \\
\hline $10-14$ & 25 \\
\hline $15-19$ & 20 \\
\hline $20-24$ & 10 \\
\hline
\end{tabular}

1. ¿Qué tipo de variable es "años de servicio"?

a) Ordinal

b) Cuantitativa de intervalo

c) Cuantitativa de ratio

2. Al introducir esta variable en SPSS, en la parte de "vista de variable" en la columna "escala", ¿cuál de las alternativas posibles se elegiría?

a) Escala

b) Ordinal

c) Nominal

3. ¿Qué gráfica es la más adecuada para representar estos datos?

a) Diagrama de sectores

b) Poligono de frecuencias

c) Gráfica de barras

4. La frecuencia relativa asociada al intervalo 15-19 es:

a) 0,13

b) 0,33

c) 0,93

5. ¿Cuál es la frecuencia acumulada para el intervalo 5-9?

Jornadas de Formación e Innovación Docente del Profesorado | № 1 (2018) Esta obra se distribuye con la licencia Creative Commons 
a) No tiene sentido calcular las frecuencias relativas acumuladas en este tipo de variables

b) 0,33

c) 0,63

6. La moda es:

a) 2

b) 7

c) 50

7. La mediana es:

a) 7,5

b) 6,5

c) 14,5

8. La media aritmética de años de servicio es:

a) 8,67

b) 13

c) 260

9. ¿Cuál es el índice de tendencia central más apropiado para esta variable?
a) Moda
b) Mediana
c) Media

10. ¿Cuál es el valor de X que deja tras de sí al $80 \%$ de los participantes?
a) 9,5
b) 14,5
c) 13

Se trabajaron en clase los contenidos a tratar todos los días. Se evaluó la asistencia a clase (entendiéndose que si no asistían, ésta podía ser la causa de que no hubiera mejora, en su caso).

Se esperaba que, aunque los contenidos fueran siendo más complejos, se mantuviera el nivel gracias al trabajo 
continuado del alumnado, que iría gradualmente aumentando sus competencias en la materia.

\section{Cuestionario inicial - final}

En el Anexo se presenta el cuestionario que se aplicó antes y después del Ciclo de Mejora Docente para comparar la evolución a lo largo del tiempo. Está conformado por las preguntas-problema asociadas a los diez contenidos que se pretendían transmitir. Con las respuestas obtenidas en estas preguntas antes de la intervención, se recogieron las ideas iniciales del alumnado. Éstas se clasificaron, desde las más imprecisas a las más apropiadas, formando así la escalera de aprendizaje (Rivero y Porlán, 2017). Se detectó la frecuencia absoluta de alumnado que se ubicó en cada una de las respuestas (para cada pregunta). Se esperaba que la frecuencia de alumnado que se ubicara en las respuestas más correctas y completas aumentara sustancialmente al comparar con el pre-test.

\section{Aplicación del Ciclo de Mejora Docente}

\section{Relato de las sesiones}

Una sesión prototípica comenzaba con la presentación de las pruebas formato cerrado a realizar, disponible en la web de la asignatura. Se dejaban unos minutos para que, en grupos de tres, el alumnado tratara de responder a la primera pregunta, argumentando la razón por la que se eligió la alternativa propuesta. Posteriormente, se contaba el número de grupos que había elegido cada alternativa. A continuación, el profesor decía la alternativa de respuesta correcta. De este modo, podía tenerse una idea aproximada de la dificultad de la pregunta (en función del porcentaje de grupos que alzaron la mano ante la respuesta que era 
correcta). De entre los grupos que habían acertado, el profesor pedía uno voluntario para explicar a sus compañeros la razón de la respuesta. Cuando llegar a la respuesta implicaba realizar cálculos, algún miembro del grupo voluntario salía a la pizarra y los hacía, mientras los demás miembros del mismo grupo explicaban los pasos que se estaban realizando en los cálculos. Se dejaba tiempo para que cualquiera que tuviera alguna duda pudiera formularla. El resto de alumnado trataba de responderla. Sólo en el caso de que el alumnado no supiera responder, el profesor intervenía. En la mayoría de las ocasiones, el profesor únicamente completaba la información dada por el alumnado. Posteriormente, se pasaba por el mismo procedimiento para la siguiente pregunta, y así sucesivamente.

\section{Evaluación del aprendizaje de los estudiantes}

El control de asistencia indicó que todo el alumnado que aprobó la asignatura asistió a más del $80 \%$ de las clases, por lo que puede afirmarse que participaron de la metodología propuesta.

Se recogieron 30 cuestionarios. La Tabla 1 compara la escalera de aprendizaje (Rivero y Porlán, 2017) al inicio y al final del Ciclo de Mejora Docente.

Tabla 1. Comparativa de la escalera de aprendizaje al inicio (pre-test) y al final (post-test) del Ciclo de Mejora.

\begin{tabular}{|c|l|c|c|}
\hline aPregunta & \multicolumn{1}{|c|}{${ }^{\text {bRespuestas }}$} & \% pre & \%post \\
\hline 1. Tipos de & Los tres tipos y distinciones correctos & 9 & 15 \\
variables y & Dos tipos y distinciones correctos & 2 & 1 \\
distinción & Tres tipos correctos, distinción no clara & 4 & 5 \\
entre ellas & Tres tipos correctos, sin distinción o dis- & 7 & 7 \\
& tinción incorrecta & 2 & 1 \\
& Dos tipos, sin distinción & 6 & 1 \\
& Nada o respuesta incorrecta & & \\
\hline
\end{tabular}

Jornadas de Formación e Innovación Docente del Profesorado | № 1 (2018) Esta obra se distribuye con la licencia Creative Commons 


\begin{tabular}{|c|c|c|c|}
\hline $\begin{array}{l}\text { 2. SPSS, in- } \\
\text { troducción } \\
\text { de datos }\end{array}$ & $\begin{array}{l}\text { Definición correcta de línea, columna y } \\
\text { procedimiento } \\
\text { Definición correcta de la línea o de la } \\
\text { columna, y del procedimiento } \\
\text { Definición correcta de la línea y de la } \\
\text { columna } \\
\text { Definición correcta de la línea o de la } \\
\text { columna } \\
\text { Nada o respuesta incorrecta }\end{array}$ & $\begin{array}{c}1 \\
0 \\
1 \\
1 \\
27\end{array}$ & $\begin{array}{l}2 \\
5 \\
8 \\
8 \\
7\end{array}$ \\
\hline $\begin{array}{l}\text { 3. Gráfi- } \\
\text { cas para } \\
\text { cada tipo de } \\
\text { variables }\end{array}$ & $\begin{array}{l}\text { Todo correcto ( } 6 \text { en total) } \\
\text { Una incorrecta o falta una } \\
\text { Una incorrecta y falta otra, o faltan dos } \\
\text { Tres correctas, tres incorrectas } \\
\text { Todo incorrecto menos una } \\
\text { Nada }\end{array}$ & $\begin{array}{l}0 \\
0 \\
0 \\
0 \\
4 \\
26\end{array}$ & $\begin{array}{l}5 \\
7 \\
6 \\
4 \\
4 \\
4\end{array}$ \\
\hline $\begin{array}{l}\text { 4. Frecuen- } \\
\text { cia rela- } \\
\text { tiva: uso y } \\
\text { cálculo }\end{array}$ & $\begin{array}{l}\text { Utilidad y cálculo correctos } \\
\text { Utilidad correcta, cálculo incorrecto } \\
\text { Cálculo correcto, sin explicación de utili- } \\
\text { dad o explicación incorrecta } \\
\text { Vaga explicación de utilidad, no cálculo } \\
\text { Nada o respuesta incorrecta }\end{array}$ & $\begin{array}{c}0 \\
0 \\
1 \\
1 \\
28\end{array}$ & $\begin{array}{c}20 \\
4 \\
5 \\
0 \\
1\end{array}$ \\
\hline $\begin{array}{l}\text { 5. Frecuen- } \\
\text { cia relativa } \\
\text { acumu- } \\
\text { lada: uso y } \\
\text { cálculo }\end{array}$ & $\begin{array}{l}\text { Utilidad y cálculo correctos } \\
\text { Utilidad correcta, cálculo incorrecto o } \\
\text { sin cálculo } \\
\text { Cálculo correcto, sin explicación de utili- } \\
\text { dad o explicación incorrecta } \\
\text { Nada o respuesta incorrecta }\end{array}$ & $\begin{array}{c}1 \\
2 \\
26\end{array}$ & $\begin{array}{c}7 \\
5 \\
14 \\
4\end{array}$ \\
\hline $\begin{array}{l}\text { 6. Moda: uso } \\
\text { y cálculo }\end{array}$ & $\begin{array}{l}\text { Utilidad y cálculo correctos } \\
\text { Utilidad correcta, cálculo incorrecto o } \\
\text { sin cálculo } \\
\text { Cálculo correcto, utilidad no explicada } \\
\text { Nada o respuesta incorrecta }\end{array}$ & $\begin{array}{c}5 \\
18 \\
0 \\
7\end{array}$ & $\begin{array}{c}18 \\
10 \\
1 \\
1\end{array}$ \\
\hline $\begin{array}{c}\text { 7. Me- } \\
\text { diana: uso y } \\
\text { cálculo }\end{array}$ & $\begin{array}{l}\text { Utilidad correcta, cálculo incompleto } \\
\text { Utilidad correcta, cálculo incorrecto o } \\
\text { sin cálculo } \\
\text { Cálculo incompleto, utilidad no } \\
\text { explicada } \\
\text { Nada }\end{array}$ & $\begin{array}{c}3 \\
11 \\
0 \\
16\end{array}$ & $\begin{array}{c}18 \\
9 \\
3 \\
0\end{array}$ \\
\hline $\begin{array}{l}\text { 8. Me- } \\
\text { dia: uso y } \\
\text { cálculo }\end{array}$ & $\begin{array}{l}\text { Utilidad y cálculo correctos } \\
\text { Utilidad correcta, cálculo incorrecto o } \\
\text { sin cálculo } \\
\text { Cálculo correcto, utilidad no explicada o } \\
\text { incorrecta } \\
\text { Nada }\end{array}$ & $\begin{array}{c}7 \\
8 \\
5 \\
10\end{array}$ & $\begin{array}{c}16 \\
3 \\
11 \\
0\end{array}$ \\
\hline
\end{tabular}

Jornadas de Formación e Innovación Docente del Profesorado | № 1 (2018) Esta obra se distribuye con la licencia Creative Commons 
Nota. 'Las preguntas se presentan de modo abreviado. La versión completa (preguntas originales) puede consultarse en el Anexo. 'Las diferentes respuestas para cada pregunta se presentan en orden, de la más completa a la más errónea.

Lo más positivo en cuestiones del alumnado fue la participación: (a) en relación a las pruebas pre-test y posttest, de los 34 matriculados en el grupo, 30 respondieron; y (b) en cuanto la asistencia a clase, 32 de los 34 matriculados estuvieron presente en más del $80 \%$ de las clases ( 6 personas acudieron al $100 \%$ de las clases).

En cuanto al conocimiento adquirido, tal y como se puede apreciar en la Tabla 1, en todas las preguntas se dio una mejora al compararse las frecuencias de respuesta antes y después del Ciclo de Mejora. En ocasiones, cuando la pregunta era muy difícil para el grupo en general, en el pre-test la mayoría no respondía nada o la respuesta era incorrecta y en el post-test se distribuían entre las respuestas algo mejores (véanse por ejemplo las preguntas 2, 3, 4, 5 y 7; cabe destacar que nadie dio la respuesta más completa posible a la pregunta 7 sobre la mediana). Excepcionalmente, en la pregunta 4, el cambio pareció ser más acentuado: mientras que al inicio muchos respondieron incorrectamente y que no lo sabían, tras el Ciclo la mayoría dieron la respuesta más completa. En general también puede decirse que, aunque aprendieron, podrían haber aprendido más, puesto que muchas veces las respuestas que dieron tras el Ciclo no fueron las más completas.

Del curso anterior al actual, se cambió de pruebas de formato abierto a pruebas de formato cerrado en la asignatura. Incluyendo en el presente curso ejercicios para practicar de formato cerrado, se consiguió mantener la coherencia entre el tipo de trabajo a realizar en las clases y en el examen que iban a realizar. 
La razón principal para el cambio de formato fue la reducción del tiempo que el alumnado tenía que esperar para recibir feedback tras realizar alguna prueba de evaluación continua. Esto indudablemente se ha logrado (se pasó de una espera media de tres semanas a recibir los resultados el mismo día en que realizaban la prueba). Sin embargo, una cuestión que podría surgir es si el nivel de aprendizaje variaría con el cambio de formato. Se podría decir que no debería haber cambio dado que, aunque el formato de respuesta es diferente, el procedimiento que el alumnado ha de llevar a cabo para la resolución del problema es el mismo. En realidad, al no haber grupo control, sería difícil de conocer la respuesta en base a evidencias. A modo de estudio piloto, comparando las calificaciones en la asignatura del grupo del presente curso y el grupo del curso anterior, ambos en primera convocatoria, se encontraron los resultados que aparecen en la Tabla 2.

Tabla 2. Comparativa de las calificaciones obtenidas en la asignatura en el grupo de inglés del curso 2016/17 y

\begin{tabular}{|ccccc|}
\multicolumn{2}{c}{$2017 / 18}$. \\
& Frecuencia & $\%$ & Frecuencia & $\%$ \\
\hline Suspenso & 0 & 0 & 0 & 0 \\
Aprobado & 14 & 38,9 & 10 & 31,3 \\
Notable & 17 & 47,2 & 16 & 50 \\
Sobresaliente & 5 & 13,9 & 6 & 18,7 \\
TOTAL & $\mathbf{3 6}$ & $\mathbf{1 0 0}$ & $\mathbf{3 2}$ & $\mathbf{1 0 0}$ \\
\hline
\end{tabular}

Las pequeñas diferencias entre ambos cursos no son estadísticamente significativas; $x_{(0,05,2)}^{2}=0,555, p=0,778$. Podría por tanto afirmarse que el cambio de formato abierto a formato cerrado no conllevó diferencias en el conocimiento adquirido por el alumnado (ni a mejor ni a peor). 


\section{Evaluación del Ciclo de Mejora Docente}

El objetivo del Ciclo de Mejora se cumplió: la evaluación del alumnado pasó de basarse en respuestas de formato abierto a estar conformada por respuesta de formato cerrado (tipo test), con lo que se consiguió que el alumnado recibiera feedback de las pruebas realizadas el mismo día de su realización (anteriormente, tenían una media de espera de un mes cuando el examen de evaluación final no se encontraba cerca). Además, en la clase se practicó con ejercicios de este tipo en el $25 \%$ de las ocasiones aproximadamente, para entrenar al alumnado de cara a las pruebas de evaluación que iba a encontrarse. El próximo curso se espera que estos logros se mantengan e incluso se generalicen a otras asignaturas que imparto.

Como cambios a introducir para el próximo curso, propondría aumentar el porcentaje de ejercicios en clase de formato cerrado (como mínimo al 30\%); y trabajar más con el programa SPSS, dado que en la pregunta 2 del pre-post, se ha visto que venían sin mucho conocimiento, y que han acabado el curso con aún escaso conocimiento. Es un software importante, dado que será el que seguramente tengan que utilizar para realizar el TFG.

Los principios didácticos que se han seguido principalmente son: (a) Recomendaciones para la elaboración de cuestionarios (Crocker y Algina, 1986). Se han utilizado para la realización del cuestionario pre-post, para los exámenes de evaluación continua y para los ejercicios de formato cerrado con los que se ha practicado en clase. Algunas recomendaciones son: no incluir alternativas de respuesta que a su vez incluyan a otras (por ejemplo, "a y b son ciertas"); no repetir información que aparece en el enunciado también en las alternativas de respuesta; hacer que concuerde gramaticalmente enunciado y alternativas; o no utilizar dobles negaciones. (b) Interacción entre compañeros y con el 
profesor (García-Pérez y Porlán, 2017): en lugar de llevar el profesorado la "voz cantante" en clase, primero trabaja el alumnado, luego hay puesta en común donde se ayudan entre compañeros (Finkel, 2008); finalmente, sólo si hace falta, el profesorado complementa. De este modo el aprendizaje se hace más significativo y, por tanto, duradero.

\section{Referencias bibliográficas}

Barbero-García, M. I., Vila-Abad, E. y Holgado-Tello, F. (2010). Psicometría: Teoría de los tests psicológicos y educativos. Madrid, España: Sanz y Torres.

Chacón-Moscoso, S. (2017). Aprendizaje integral y aplicado en análisis de datos. Comunicación presentada en las IV Jornadas de Formación e innovación docente. Sevilla, España: ICE.

Crocker, L. M. y Algina, J. (1986). Introduction to classical and modern test theory. New York, NY: Holt, Rinehart \& Winston.

de Alba, N. y Porlán, R. (2017). La metodología de la enseñanza. En R. Porlán (Coord.), Enseñanza Universitaria. Cómo mejorarla (pp. 37-53). Madrid, España: Morata.

Finkel, D. (2008). Dar clase con la boca cerrada. Valencia, España: Universitat de València.

García-Díaz, E., Porlán, R. y Navarro, E. (2017). Los fines y los contenidos de enseñanza. En R. Porlán (Coord.), Enseñanza Universitaria. Cómo mejorarla (pp. 55-72). Madrid, España: Morata.

García-Pérez, F. y Porlán, R. (2017). Los principios didácticos y el modelo didáctico personal. En R. Porlán (Coord.), Enseñanza Universitaria. Cómo mejorarla (pp. 93-104). Madrid, España: Morata.

Rivero, A. y Porlán, R. (2017). La evaluación en la enseñanza universitaria. En R. Porlán (Coord.), Enseñanza Universitaria. Cómo mejorarla (pp. 73-91). Madrid, España: Morata. 


\begin{tabular}{|l|l|l|}
\hline \multicolumn{1}{|c|}{ QUESTION } & BEFORE STARTING THE SUBJECT & NOW \\
\hline $\begin{array}{l}\text { Which are the kinds of va- } \\
\text { riables depending on their } \\
\text { kind of measure? How } \\
\text { can I distinguish between } \\
\text { them? }\end{array}$ & & \\
\hline $\begin{array}{l}\text { Which information do you } \\
\text { include in a line in SPSS? } \\
\text { And a column? How do I } \\
\text { introduce data? }\end{array}$ & & \\
\hline $\begin{array}{l}\text { Which are the appropriate } \\
\text { graphics for each kind of } \\
\text { variable? }\end{array}$ & & \\
\hline $\begin{array}{l}\text { What is the use of the re- } \\
\text { lative frequency? How is it } \\
\text { calculated? }\end{array}$ & & \\
\hline $\begin{array}{l}\text { What is the use of } \\
\text { the cumulative rela- } \\
\text { tive frequency? How is it } \\
\text { calculated? }\end{array}$ & & \\
\hline $\begin{array}{l}\text { What is the use of } \\
\text { the mode? How is it } \\
\text { calculated? }\end{array}$ & & \\
\hline $\begin{array}{l}\text { What is the use of the me- } \\
\text { dian? How is it calculated? }\end{array}$ & & \\
\hline $\begin{array}{l}\text { What is the use of } \\
\text { the mean? How is it } \\
\text { calculated? }\end{array}$ & \\
\hline
\end{tabular}

Anexo. Cuestionario cumplimentado antes y después del Ciclo de Mejora Docente

Please, answer these questions considering what you knew before starting the course and now. 